Article

\title{
Lyapunov Characterization of the Fractional Nonlinear Systems with Exogenous Input
}

\author{
Ndolane Sene \\ Laboratoire Lmdan, Département de Mathématiques de la Décision, Université Cheikh Anta Diop de Dakar, \\ BP 5683 Dakar Fann, Senegal; ndolane.sene@ucad.edu.sn \\ Received: 31 March 2018; Accepted: 27 April 2018; Published: 2 May 2018 \\ check for \\ updates
}

\begin{abstract}
This paper deals with a Lyapunov characterization of the conditional Mittag-Leffler stability and conditional asymptotic stability of the fractional nonlinear systems with exogenous input. A particular class of the fractional nonlinear systems is studied. The paper contributes to giving in particular the Lyapunov characterization of fractional linear systems and fractional bilinear systems with exogenous input.
\end{abstract}

Keywords: Riemann-Liouville derivative; fractional nonlinear systems; conditional asymptotic stability

\section{Introduction}

Fractional calculus has been extensively studied in recent years and many results have been given: Baleanu et al. in [1], Caputo and Fabrizio in [2], Atangana et al. in [3], Podlubny in [4], Torres and Malinowska in [5], Kilbas et al. in [6], Petras in [7], and others. In the last decade the fractional calculus mainly received attention due to its important role in modeling the anomalous dynamics of various processes related to complex systems in most areas of science and engineering. Fractional differential equations have found many applications in physics, control engineering and signal processing [8-13]. It was found that many systems in interdisciplinary fields can be elegantly described with the help of the fractional derivative. Various types of the fractional derivatives were introduced in the literature such as the Riemann-Liouville derivative, Caputo derivative, Caputo and Fabrizio derivative, Atangana-Baleanu-Riemann derivative, Atangana-Baleanu-Caputo derivative, etc. It is well known that many of them verify Leibniz property. The fractional derivative has a long history. In 1695, l'Hospital asked a remarkable question, what does it mean $\frac{d^{n} f}{d x^{n}}$ when $n=\frac{1}{2}$ [14]. The limit definition of a fractional derivative was introduced to answer this question [14,15]. Fractional calculus is a generalization of ordinary differential and integration to arbitrary non integer order. In $[4,16]$ Riemann-Liouville proposed alternative of the definition of the fractional derivative expressed as follows

$$
D_{\alpha}^{R L} f(t)=\frac{1}{\Gamma(1-\alpha)} \frac{d}{d t} \int_{0}^{t}(t-s)^{\alpha-1} f(s) d s
$$

In the literature, many contributions exist using the Riemann-Liouville derivative in many areas of science.

In 2014, in [14], Khalil gives a definition of a derivative called conformable derivative mathematically expressed by

$$
T_{\alpha} f(t)=\lim _{\epsilon \rightarrow 0} \frac{f\left(t+\epsilon t^{1-\alpha}\right)-f(t)}{\epsilon}
$$


In [17], Almeida introduced a similar limit definition of fractional derivative of a function if we do not know the kernel as follows

$$
f^{\alpha}(t)=\lim _{\epsilon \rightarrow 0} \frac{f\left(t+\epsilon k(t)^{1-\alpha}\right)-f(t)}{\epsilon} .
$$

If the $k(t)=t$, we recover the conformable derivative given by Khalil. If expanding the function $t e^{\epsilon t^{1-\alpha}}$ at neighborhood of $\epsilon=0$ it follows that $t e^{\epsilon t^{1-\alpha}}=t+\epsilon t^{1-\alpha}+o(\epsilon)$, we recover the Katugampola idea of fractional derivative given in [18] expressed as

$$
D_{\alpha} f(t)=\lim _{\epsilon \rightarrow 0} \frac{f\left(t e^{\epsilon t^{1-\alpha}}\right)-f(t)}{\epsilon} .
$$

In 2015, Caputo and Fabrizio introduced new fractional derivative defined as follows

$$
D_{\alpha}^{C F} f(t)=\frac{(2-\alpha) M(\alpha)}{2(1-\alpha)} \int_{0}^{t} \exp \left(-\frac{\alpha}{1-\alpha}(t-s)\right) f^{\prime}(s) d s
$$

Another definition to generalize this definition is proposed by Baleanu and Atangana in [1]. We can see with this above definition if $\alpha \rightarrow 1$, we recover the classical derivative [2]. In this paper, we will use the Capoto idea of the fractional derivative given by the condition (5). In [16] the author proposes a condition for the stability of the fractional differential equations using the Riemann-Liouville derivative. In [19] the author uses the conformable derivative to find conditions for the stability for a particular class of the fractional nonlinear systems. In these contributions the authors give conditions on the stability using the Lyapunov direct method. In this paper, we analyze new stability notions for the fractional differential equations with exogenous input called conditional Mittag-Leffler stability and conditional asymptotic stability. Stability of nonlinear systems received increased attention due to its important role in areas of science and engineering. A large number of monograph and papers are devoted to the fractional nonlinear systems $[8,20,21]$. This paper deals with the stability notions of the fractional nonlinear systems with input using Caputo derivative. It treats in particular the conditional Mittag-Leffler stability and the conditional asymptotic stability of a particular class of the fractional nonlinear systems with input using the Caputo derivative. Many results in stability analysis of the fractional nonlinear systems are consigned in [21-26]. In [20], author study stability analysis of a particular class of the fractional nonlinear systems using the Lyapunov direct method. Note that Lyapunov indirect method can be applied in the context of fractional systems. We have to get the Jacobian matrix and apply the results in [16] to reach a conclusion on the stability.

It is well known of fractional linear differential equations who states matrix are Hurwitz or verify the condition $\mid \arg \left(\lambda(A) \mid>\frac{\alpha \pi}{2}\right.$ are asymptotically stable. Stability condition for FDE linear time delayed have already been obtained. Recently Delavari et al. [22] have done elegant works in the stability of fractional nonlinear systems. For the fractional nonlinear systems with conformable derivative, it is known if the state matrix is Hurwitz we have the asymptotic stability or the fractional exponential stability (see [19]). Providing conditions under which the fractional nonlinear systems with exogenous input using Caputo derivative are conditional Mittag-Leffler stable and conditional asymptotically stable have been the object of intense research.

The paper is organized as follows: in Section 2, after recalling some necessary definitions, we will describe the class of the fractional nonlinear systems, and will provide the main results. In Section 3, we will give the numerical example which motives this work and illustrate our main results. Then, we are going to end this paper by giving our proofs, conclusions and remarks in Section 4 .

Notation 1. PD denotes the set of all continuous functions $\chi: \mathbb{R}_{\geq 0} \rightarrow \mathbb{R}_{\geq 0}$ satisfying $\chi(0)=0$ and $\chi(s)>0$ for all $s>0$. A class $\mathcal{K}$ function is an increasing $\mathcal{P D}$ function. The class $\mathcal{K}_{\infty}$ denotes the set of all unbounded $\mathcal{K}$ function. A continuous function $\beta: \mathbb{R}_{\geq 0} \times \mathbb{R}_{\geq 0} \rightarrow \mathbb{R}_{\geq 0}$ is said to be class $\mathcal{K} \mathcal{L}$ if $\beta($., $t) \in \mathcal{K}$ 
for any $t \geq 0$ and $\beta(s,$.$) is non increasing and tends to zero as its arguments tends to infinity. Given x \in \mathbb{R}^{n}$, $\|x\|$ stands for its Euclidean norm: $\|x\|:=\sqrt{x_{1}^{2}+\ldots+x_{n}^{2}}$. For a matrix $A, \lambda_{\text {max }}(A)$ and $\lambda_{\text {min }}(A)$ denote the maximal and the minimal eigenvalue of $A$, respectively. If the condition $\operatorname{Re}\left(\lambda_{i}\right)<0, \forall i=1,2, \ldots, n$, holds then the matrix $A$ is said Hurwitz.

\section{Preliminaries Definitions and Main Results}

\subsection{Preliminaries Definitions}

In this section, we introduce some definitions of the fractional calculus and several lemmas which we will use later.

Definition $1([16,27,28])$. Given a function $f:[0,+\infty[\longrightarrow \mathbb{R}$. Then the Riemann-Liouville fractional derivative of $f$ of order $\alpha$ is defined by

$$
D_{\alpha}^{R L} f(t)=\frac{1}{\Gamma(1-\alpha)} \frac{d}{d t} \int_{0}^{t}(t-s)^{\alpha-1} f(s) d s
$$

all $t>0, \alpha \in(0,1)$.

Definition $2([16,27,29])$. Given a function $f:[0,+\infty[\longrightarrow \mathbb{R}$. Then the Caputo fractional derivative of $f$ of order $\alpha$ is defined by

$$
D_{\alpha}^{c} f(t)=\frac{1}{\Gamma(1-\alpha)} \int_{0}^{t} \frac{f^{\prime}(s)}{(t-s)^{\alpha}} d s
$$

all $t>0, \alpha \in(0,1)$.

Definition 3 ([29-31]). Given a function $f:[0,+\infty[\longrightarrow \mathbb{R}$. Then the Riemann-Liouville integral of $f$ of order $\alpha$ is defined by

$$
I_{\alpha}^{R L} f(t)=\frac{1}{\Gamma(\alpha)} \int_{0}^{t}(t-s)^{\alpha-1} f(s) d s
$$

all $t>0, \alpha \in(0,1)$.

It is well known that Riemann-Liouville and Caputo derivatives are used to verify the Leibniz property. For Riemann-Liouville fractional integrals and derivatives, the following composition rule is held:

Lemma 1 ([32]). Let $\alpha \in(0,1]$ and $f$ is any continuous function in a domain of $I_{\alpha}$, for $t>0$ we have

$$
I_{\alpha}^{R L} \circ D_{\alpha}^{R L} f(t)=f(t) .
$$

The Mittag-Leffler function is a generalization of the first order exponential and occurs at a fundamental place on the stability analysis of the fractional differential equations. We have the following definition.

Definition $4([33,34])$. The two-parameters Mittag-Leffler function is defined as

$$
E_{\alpha, \beta}(z)=\sum_{k=0}^{\infty} \frac{z^{k}}{\Gamma(\alpha k+\beta)}
$$

where $\alpha>0$ and $\beta>0$.

The classical exponential $E_{1,1}(z)=\exp (z)$ is obtained by taking $\alpha=1$ and $\beta=1$. For more information on Mittag-Leffler functions see [35]. 
Now let us obtain the solution of the following fractional differential equation, whose solution is fundamental for our main results of this paper. The form of the solution of the equation described in the following lemma is well known in the literature.

Lemma 2 ([31]). Let $\alpha \in(0,1)$. Then the unique solution of the following initial value problem

$$
D_{\alpha}^{c} f(t)=\lambda f(t)+m(t)
$$

is given by

$$
f(t)=f\left(t_{0}\right) E_{\alpha}\left(\lambda\left(t-t_{0}\right)^{\alpha}\right)+\int_{t_{0}}^{t}(t-s)^{\alpha-1} E_{\alpha, \alpha}\left(\lambda(t-s)^{\alpha}\right) m(s) d s
$$

As follows we give the Gronwall lemma which plays an important role in stability analysis of the fractional nonlinear systems.

Lemma 3 (Gronwall Inequality [16]). Suppose that $g(t)$ and $u(t)$ are continuous functions on the interval in $\left[t_{0}, t\right]$ such that $g(t) \geq 0$ and $u(t) \geq 0$ and satisfies the following condition

$$
u(t) \leq \lambda+\int_{t_{0}}^{t}[g(s) u(s)+r] d s
$$

Then it holds that

$$
u(t) \leq\left(\lambda+r\left(t-t_{0}\right)\right) \exp \left(\int_{t_{0}}^{t} g(s) d s\right)
$$

where $\lambda \geq 0$ and $r \geq 0$ are two positives constants.

Generally the fractional nonlinear systems with exogenous input which we consider are mathematically represented by the following form

$$
D_{\alpha}^{c} x(t)=f(t, x(t), u(t))
$$

where $x(t) \in \mathbb{R}^{n}$ is state variable, $u \in \mathbb{R}^{m}$ represents the exogenous input and $f: \mathbb{R}^{+} \times \mathbb{R} \rightarrow \mathbb{R}^{n}$ a continuous locally Lipschitz function satisfying $f(t, 0,0)=0$ for all $t>0$. Given initial condition $x_{0} \in \mathbb{R}^{n}$ and an input $u \in \mathbb{R}^{m}$, the solution of (14) starting at $x_{0}$ at time $t=0$ is denoted by $x(t)=x\left(., x_{0}, u\right)$.

Because the function $f$ is continuous and locally Lipschitz, the solutions of the fractional differential system defined by (14) exist. The existence of the solution of the fractional nonlinear system is important before doing the stability analysis.

We introduce some definitions of the fractional nonlinear system (14).

Definition 5 ([21]). The trivial solution of system $D_{\alpha}^{c} x(t)=f(t, x, 0)$ is said to be stable if for every $\epsilon>0$ there exists a $\delta=\delta(\epsilon)$ such that for any initial condition $\left\|x\left(t_{0}\right)\right\|<\delta$, the solution $x(t)$ of the system $D_{\alpha}^{c} x(t)=f(t, x, 0)$ satisfies inequality $\|x(t)\|<\epsilon$ for all $t>t_{0}$.

The trivial solution of system $D_{\alpha}^{c} x(t)=f(t, x, 0)$ is said to be asymptotically stable if it is stable and furthermore $\lim _{t \rightarrow+\infty} x(t)=0$.

For the main results of this paper, we introduce new definitions on the stability analysis of the fractional calculus.

Definition 6. The fractional nonlinear system defined by (14) is said to be conditional Mittag-Leffler stable if for $\epsilon>0$ such that for any input satisfying $\|u\| \leq \epsilon$, for any bounded initial condition $\left\|x\left(t_{0}\right)\right\|$ its solution satisfies

$$
\left\|x\left(t, x_{0}, u\right)\right\| \leq\left[K\left\|x\left(t_{0}\right)\right\| E_{\alpha}\left(\lambda\left(t-t_{0}\right)^{\alpha}\right)\right]^{\frac{1}{b}}
$$


where $b>0, K>0$.

Definition 7. The fractional nonlinear system defined by (14) is said to be conditional asymptotic stable if for $\epsilon>0$ such that for any input satisfying $\|u\| \leq \epsilon$, there exist a class $\mathcal{K} \mathcal{L}$ function $\beta$ such that for any bounded initial condition $\left\|x\left(t_{0}\right)\right\|$ its solution satisfies

$$
\left\|x\left(t, x_{0}, u\right)\right\| \leq \beta\left(\left\|x\left(t_{0}\right)\right\|, t-t_{0}\right)
$$

\subsection{Main Results}

We are now ready to state the main results of this paper which are provided in Section 4. Generally the fractional nonlinear systems which we consider are mathematically represented by the following form

$$
D_{\alpha}^{c} x(t)=f(t, x(t), u(t))=A x(t)+u B x(t)
$$

where $x(t) \in \mathbb{R}^{n}$ is state variable, $A$ is an matrix in $\mathbb{R}^{n \times n}, B$ is an matrix in $\mathbb{R}^{n \times n}$ and $u \in \mathbb{R}$ the exogenous input.

The fractional nonlinear system defined by (17) is called the fractional bilinear system with exogenous input. We make the following result.

Theorem 1. Let $x=0$ be an equilibrium point of the system (17). If the state matrix $A$ with $\alpha \in(0,1)$, satisfies the condition $\mid \arg \left(\lambda(A) \mid>\frac{\alpha \pi}{2}\right.$ and the exogenous input bounded $\|u\| \leq \epsilon$ then the trivial solution of the fractional linear system (17) is conditional asymptotically stable.

Before finishing the main result of this section we give some Lyapunov characterization of the conditional Mittag-Leffler stability and it is conditional asymptotically stable.

Theorem 2. Let $x=0$ be an equilibrium point for the fractional nonlinear system (14) and there exists a positive function $V: \mathbb{R}^{+} \times \mathbb{R}^{n} \longrightarrow \mathbb{R}$ be continuous, and a class $\mathcal{K}_{\infty}$ function $\gamma$ satisfying following conditions

1. $\|x(t)\|^{a} \leq V(t, x(t))$

2. $V(t, x)$ has Caputo fractional derivative of order $\alpha$ for all $t_{0} \geq 0$

3. $D_{\alpha}^{c} V(t, x(t)) \leq-(c-\gamma(\|u\|)) V(t, x(t))$ with $c>\gamma(\|u\|)$

where $c$ and a are non negative constants. If the exogenous input is bounded, then the origin of the fractional nonlinear system (14) is conditional Mittag-Leffler stable.

For the conditional asymptotic stability of fractional nonlinear stable, we make the following theorems.

Theorem 3. Let $x=0$ be an equilibrium point for the fractional nonlinear system (14) and there exist a positive function $V: \mathbb{R}^{+} \times \mathbb{R}^{n} \longrightarrow \mathbb{R}$ be continuous, and a class $\mathcal{K}$ functions $\chi_{2}, \chi_{3}$ and $\gamma \in \mathcal{K}_{\infty}$ satisfying following conditions

1. $\chi_{2}(\|x\|) \leq V(t, x) \leq \chi_{3}(\|x\|)$.

2. $V(t, x)$ has caputo fractional derivative of order $\alpha$ for all $t_{0} \geq 0$

3. $D_{\alpha}^{c} V(t, x(t)) \leq-(c-\gamma(\|u\|)) V(t, x(t))$ with $c>\gamma(\|u\|)$

where $c$ is non negative constant. If the exogenous input is bounded, then the origin of the fractional nonlinear system (14) is conditional asymptotically stable.

Theorem 4. Let $x=0$ be an equilibrium point for the fractional nonlinear system (14) and there exist a positive function $V: \mathbb{R}^{+} \times \mathbb{R}^{n} \longrightarrow \mathbb{R}$ be continuous, and a class $\mathcal{K}$ functions $\chi_{2}, \chi_{3}, \chi_{4}$ and $\gamma \in \mathcal{K}_{\infty}$ satisfying following conditions

1. $\chi_{2}(\|x\|) \leq V(t, x) \leq \chi_{3}(\|x\|)$. 
2. $V(t, x)$ has caputo fractional derivative of order $\alpha$ for all $t_{0} \geq 0$

3. $D_{\alpha}^{c} V(t, x(t)) \leq-(c-\gamma(\|u\|)) \chi_{4}(\|x\|)$ with $c>\gamma(\|u\|)$

where $c$ is non negative constant. If the exogenous input is bounded, then the origin of the fractional nonlinear system (14) is conditional asymptotically stable.

These main results give some new results on the stability analysis of the fractional nonlinear systems with exogenous input and can be used to find other stability notions.

\section{Motivation and Numerical Example}

In this section, two examples are provided to illustrate the Lyapunov characterization given in the previous section. For an illustration of our results, let the following fractional bilinear system defined as

$$
D_{\alpha}^{c} x(t)=A x(t)+u B x(t)
$$

where $x(t) \in \mathbb{R}^{n}$ is state variable, $A$ is an Hurwitz matrix in $\mathbb{R}^{n \times n}, B$ is an matrix in $\mathbb{R}^{n \times n}$ and $u \in \mathbb{R}$ the exogenous input. We choose a Lyapunov candidate function $V(t, x(t))=x^{T} P x$ where $A^{T} P+P A=-Q$. The $\alpha$ derivative of $V$ along the trajectories of (17) is given by

$$
\begin{aligned}
D_{\alpha}^{c} V(t, x(t)) \leq 2 x^{T} P D_{\alpha}^{c} x & =[A x+u B x]^{T} P x+x^{T} P[A x+u B x] \\
& =x^{T} A^{T} P x+(u B x)^{T} P x+x^{T} P A x+x^{T} P(u B x) \\
& =x^{T}\left(A^{T} P+P A\right) x+(u B x)^{T} P x+x^{T} P(u B x) \\
& \leq-\lambda_{\min }(Q)\|x\|^{2}+2 \lambda_{\max }(P)\|u\|\|B\|\|x\|^{2} \\
& =-\left[\lambda_{\min }(Q)-2 \lambda_{\max }(P)\|u\|\|B\|\right]\|x\|^{2}
\end{aligned}
$$

It follows by Theorem 4 , if the input satisfies the following condition $\lambda_{\min }(Q)-$ $2 \lambda_{\max }(P)\|u\|\|B\|>0$, then the trivial solution of the fractional nonlinear system (18) with the exogenous input is conditional asymptotically stable. This example has particularly motivated the works in this paper.

For numerical example, let us consider the fractional bilinear system

$$
D_{\alpha}^{c} x=-x+u x
$$

where $x \in \mathbb{R}$ and $u \in \mathbb{R}$ the exogenous input. Let the Lyapunov candidate function be definite by $V(t, x(t))=\frac{1}{2} x^{2}$. The $\alpha$-derivative of $V$ along the trajectory of (19) is given by

$$
\begin{aligned}
D_{\alpha}^{c} V(t, x(t)) \leq x^{T} D_{\alpha}^{c} x & =x(-x+u x) \\
& =-x^{2}+u x^{2} \\
& =-(2-2 u) V(t, x)
\end{aligned}
$$

It follows from Theorem 3, if the exogenous input satisfies the following condition $\|u\|<1$ (condition for the stability), then the trivial solution of the fractional nonlinear system (19) with the exogenous input is conditional asymptotically stable.

\section{Proofs of the Theorems}

\subsection{Proof of Theorem 1}

The solution of the fractional differential Equation (17) using Lemma 2 is given by

$$
x(t)=x\left(t_{0}\right) E_{\alpha}\left(A\left(t-t_{0}\right)^{\alpha}\right)+\int_{t_{0}}^{t}(t-s)^{\alpha-1} E_{\alpha, \alpha}\left(A(t-s)^{\alpha}\right) u B x(s) d s
$$


From which it follows that the following inequality

$$
\|x(t)\| \leq\left\|x\left(t_{0}\right)\right\|\left\|E_{\alpha}\left(A\left(t-t_{0}\right)^{\alpha}\right)\right\|+\|B\| \int_{t_{0}}^{t}(t-s)^{\alpha-1} E_{\alpha, \alpha}\left(A(t-s)^{\alpha}\right)\|u\|\|x(s)\| d s .
$$

From the fact that the input bounded $\|u\|<\epsilon$, we have the following inequality

$$
\|x(t)\| \leq\left\|x\left(t_{0}\right)\right\|\left\|E_{\alpha}\left(A\left(t-t_{0}\right)^{\alpha}\right)\right\|+\|B\| \epsilon \int_{t_{0}}^{t}(t-s)^{\alpha-1} E_{\alpha, \alpha}\left(A(t-s)^{\alpha}\right)\|x(s)\| d s .
$$

Using Gronwall lemma, it follows that the above inequality become

$$
\|x(t)\| \leq\left\|x\left(t_{0}\right)\right\|\left\|E_{\alpha}\left(A\left(t-t_{0}\right)^{\alpha}\right)\right\| \exp \left\{\|B\| \epsilon \int_{t_{0}}^{t}(t-s)^{\alpha-1} E_{\alpha, \alpha}\left(A(t-s)^{\alpha}\right) d s\right\} .
$$

If the state matrix $A$ satisfies the condition $\mid \arg \left(\lambda(A) \mid>\frac{\alpha \pi}{2}\right.$, according to [16] there exist a positive constant $M$ such that

$$
\left.\int_{t_{0}}^{t}(t-s)^{\alpha-1} E_{\alpha, \alpha}\left(A(t-s)^{\alpha}\right)\right) d s \leq M
$$

Then we have the following inequality

$$
\|x(t)\| \leq\left\|x\left(t_{0}\right)\right\|\left\|E_{\alpha}\left(A\left(t-t_{0}\right)^{\alpha}\right)\right\| \exp \{\|B\| \epsilon M\} .
$$

With $\left.\beta\left(\left\|x_{0}\right\|, t-t_{0}\right)\right)=\left\|x\left(t_{0}\right)\right\|\left\|E_{\alpha}\left(A\left(t-t_{0}\right)^{\alpha}\right)\right\| \exp \{\|B\| \epsilon M\}$, that end the proof of the Theorem.

\subsection{Proof of Theorem 2}

Let $x=0$ be an equilibrium point for the fractional nonlinear system (14) and there exists a positive function $V: \mathbb{R}^{+} \times \mathbb{R}^{n} \longrightarrow \mathbb{R}$ be continuous, and a class $\mathcal{K}_{\infty}$ function $\gamma$ satisfying following conditions

1. $\|x(t)\|^{a} \leq V(t, x(t))$

2. $V(t, x)$ has Caputo fractional derivative of order $\alpha$ for all $t_{0} \geq 0$

3. $D_{\alpha}^{c} V(t, x(t)) \leq-(c-\gamma(\|u\|)) V(t, x(t))$ with $c>\gamma(\|u\|)$

By assumption that $\|u\| \leq \epsilon$, we obtain using the condition (3)

$$
D_{\alpha}^{c} V(t, x(t)) \leq-(c-\gamma(\epsilon)) V(t, x(t)) .
$$

We know that there exists a positive continuous function $M(t)$ such that

$$
D_{\alpha}^{c} V(t, x(t))=-k V(t, x(t))-M(t) .
$$

where $k=c-\gamma(\epsilon)$. Using the Lemma 2, we have the following equality

$$
V(t, x)=V\left(t_{0}, x_{0}\right) E_{\alpha}\left(-k\left(t-t_{0}\right)^{\alpha}\right)-\int_{t_{0}}^{t}(t-s)^{\alpha-1} E_{\alpha, \alpha}\left(-k(t-s)^{\alpha}\right) M(s) d s
$$

With the fact that $\int_{t_{0}}^{t}(t-s)^{\alpha-1} E_{\alpha, \alpha}\left(-k(t-s)^{\alpha}\right) M(s) d s \geq 0$. We obtain the following inequality given by

$$
V(t, x) \leq V\left(t_{0}, x_{0}\right) E_{\alpha}\left(-k\left(t-t_{0}\right)^{\alpha}\right)
$$

Now using the first condition, it follows that the following inequality

$$
\|x(t)\|^{a} \leq V(t, x) \leq V\left(t_{0}, x_{0}\right) E_{\alpha}\left(-k\left(t-t_{0}\right)^{\alpha}\right)
$$


Then

$$
\|x(t)\| \leq\left[V\left(t_{0}, x_{0}\right) E_{\alpha}\left(-k\left(t-t_{0}\right)^{\alpha}\right)\right]^{\frac{1}{a}}
$$

Then the origin of the fractional nonlinear system (14) is conditional Mittag-Leffler stable.

\subsection{Proof of Theorem 3}

Let $x=0$ be an equilibrium point for the fractional nonlinear system (14) and there exists a positive function $V: \mathbb{R}^{+} \times \mathbb{R}^{n} \longrightarrow \mathbb{R}$ be continuous, and a class $\mathcal{K}$ functions $\chi_{2}, \chi_{3}$ and $\gamma \in \mathcal{K}_{\infty}$ satisfying following conditions

1. $\chi_{2}(\|x\|) \leq V(t, x) \leq \chi_{3}(\|x\|)$.

2. $V(t, x)$ has Caputo fractional derivative of order $\alpha$ for all $t_{0} \geq 0$

3. $D_{\alpha}^{c} V(t, x(t)) \leq-(c-\gamma(\|u\|)) V(t, x(t))$ with $c>\gamma(\|u\|)$

By assumption that $\|u\| \leq \epsilon$, we obtain using the condition (3)

$$
D_{\alpha}^{c} V(t, x(t)) \leq-(c-\gamma(\epsilon)) V(t, x(t)) .
$$

We know that there exist a positive continuous function $M(t)$ such that

$$
D_{\alpha}^{c} V(t, x(t))=-k V(t, x(t))-M(t) .
$$

where $k=c-\gamma(\epsilon)$. Using the Lemma (2), we have the following equality

$$
V(t, x)=V\left(t_{0}, x_{0}\right) E_{\alpha}\left(-k\left(t-t_{0}\right)^{\alpha}\right)-\int_{t_{0}}^{t}(t-s)^{\alpha-1} E_{\alpha, \alpha}\left(-k(t-s)^{\alpha}\right) M(s) d s
$$

With the fact that $\int_{t_{0}}^{t}(t-s)^{\alpha-1} E_{\alpha, \alpha}\left(-k(t-s)^{\alpha}\right) M(s) d s \geq 0$. We obtain the following inequality given by

$$
V(t, x) \leq V\left(t_{0}, x_{0}\right) E_{\alpha}\left(-k\left(t-t_{0}\right)^{\alpha}\right)
$$

Recalling that $\chi_{2}^{-1}(a+b) \leq \chi_{2}^{-1}(2 a)+\chi_{2}^{-1}(2 b)$ as $\chi_{2} \in \mathcal{K}$. Then

$$
\|x(t)\| \leq \chi_{2}^{-1}\left(2 \chi_{3}\left(\left\|x_{0}\right\|\right) E_{\alpha}\left(-k\left(t-t_{0}\right)^{\alpha}\right)\right)+\chi_{2}^{-1}(0)
$$

Taking the function $\left.\beta\left(\left\|x_{0}\right\|, t-t_{0}\right)\right)=\chi_{2}^{-1}\left(2 \chi_{3}\left(x_{0}\right) E_{\alpha}\left(-k\left(t-t_{0}\right)^{\alpha}\right)\right)$ and we know $\chi_{2}^{-1}(0)=0$. We obtain that

$$
\left.\|x(t)\| \leq \beta\left(\left\|x_{0}\right\|, t-t_{0}\right)\right)
$$

Then the origin of the fractional nonlinear system (14) is conditional asymptotically stable.

\subsection{Proof of Theorem 4}

Let $x=0$ be an equilibrium point for the fractional nonlinear system (14) and there exist a positive function $V: \mathbb{R}^{+} \times \mathbb{R}^{n} \longrightarrow \mathbb{R}$ be continuous, and a class $\mathcal{K}$ functions $\chi_{2}, \chi_{3}, \chi_{4}$ and $\gamma \in \mathcal{K}_{\infty}$ satisfying following conditions

1. $\chi_{2}(\|x\|) \leq V(t, x) \leq \chi_{3}(\|x\|)$.

2. $V(t, x)$ has Caputo fractional derivative of order $\alpha$ for all $t_{0} \geq 0$

3. $D_{\alpha}^{c} V(t, x(t)) \leq-(c-\gamma(\|u\|)) \chi_{4}(\|x\|)$ with $c>\gamma(\|u\|)$

By assumption that $\|u\| \leq \epsilon$, we obtain from the conditions (1) and (3) that

$$
D_{\alpha}^{c} V(t, x(t)) \leq-(c-\gamma(\epsilon)) \chi_{4}\left(\chi_{3}^{-1}(V(t, x(t))) .\right.
$$


Let $k=c-\gamma(\epsilon)$, it follows

$$
D_{\alpha}^{c} V(t, x(t)) \leq-k \chi_{4}\left(\chi_{3}^{-1}(V(t, x(t))) .\right.
$$

Using proof of (Theorem 3.1 [22]) there exist a class $\mathcal{K} \mathcal{L}$ function $\mu$ such that

$$
V(t, x(t)) \leq \mu\left(\chi_{3}\left(x_{0}\right), t-t_{0}\right) .
$$

Using the first assumption we obtain that

$$
\chi_{2}(\|x\|) \leq \mu\left(\chi_{3}\left(x_{0}\right), t-t_{0}\right) .
$$

Recalling that $\chi_{2}^{-1}(a+b) \leq \chi_{2}^{-1}(2 a)+\chi_{2}^{-1}(2 b)$ as $\chi_{2} \in \mathcal{K}$. Then we have

$$
\|x(t)\| \leq \chi_{2}^{-1}\left(2 \mu\left(\chi_{3}\left(x_{0}\right), t-t_{0}\right)\right)+\chi_{2}^{-1}(0)
$$

Taking the function $\left.\beta\left(\left\|x_{0}\right\|, t-t_{0}\right)\right)=\chi_{2}^{-1}\left(2 \mu\left(\chi_{3}\left(x_{0}\right), t-t_{0}\right)\right) \in \mathcal{K} \mathcal{L}$ and we know $\chi_{2}^{-1}(0)=0$. We obtain that

$$
\left.\|x(t)\| \leq \beta\left(\left\|x_{0}\right\|, t-t_{0}\right)\right)
$$

Then the origin of the fractional nonlinear system (14) is conditional asymptotically stable.

\section{Conclusions}

We have discussed in this paper the conditional Mittag-Leffler stability and conditional asymptotic stability of the fractional nonlinear system with exogenous input using a Caputo derivative operator. It contributes to introducing a new stability notion for the fractional nonlinear systems with exogenous input. Comparison lemma and comparison functions have played an important role in this paper.

Acknowledgments: The author thanks both referees and managing editor of Fractal Fract for their careful reading of the manuscript, for their comments, and their corrections.

Conflicts of Interest: The author declares no conflict of interest.

\section{References}

1. Baleanu, D.; Güvenç, Z.B.; Machado, J.T. (Eds.) New Trends in Nanotechnology and Fractional Calculus Applications; Springer: Berlin, Germany, 2010.

2. Caputo, M.; Fabrizio, M. A new definition of fractional derivative without singular kernel. Progr. Fract. Differ. Appl. 2015, 1, 73-85.

3. Atangana, A.; Baleanu, D. New fractional derivatives with nonlocal and non-singular kernel: Theory and application to heat transfer model. arXiv 2016, arXiv:1602.03408.

4. Podlubny, I. Matrix approach to discrete fractional calculus II: Partial fractional differential equations. J. Comput. Phys. 2009, 228, 3137-3153. [CrossRef]

5. Malinowska, A.B.; Torres, D.F. Introduction to the Fractional Calculus of Variations; World Scientific Publishing Company: Singapore, 2012.

6. Kilbas, A.A.; Srivastava, H.M.; Trujillo, J.J. Theory and Applications of Fractional Differential Equations; Elsevier Science: New York, NY, USA, 2006.

7. Petráš, I. Fractional-Order Nonlinear Systems: Modeling, Analysis and Simulation; Springer Science \& Business Media: Berlin/Heidelberg, Germany, 2011.

8. Chung, W.S. Fractional Newton mechanics with conformable fractional derivative. J. Comput. Appl. Math. 2015, 290, 150-158. [CrossRef]

9. Eslami, M. Exact traveling wave solutions to the fractional coupled nonlinear Schrodinger equations. Appl. Math. Comput. 2016, 285, 141-148. [CrossRef]

10. Miller, K.S.; Ross, B. An Introduction to the Fractional Calculus and Fractional Differential Equations; Wiley: New York, NY, USA, 1993. 
11. Oldham, K.; Spanier, J. The Fractional Calculus Theory and Applications of Differentiation and Integration to Arbitrary Order; Elsevier: New York, NY, USA, 1974; Volume 111.

12. Laskin, N. Fractional schrödinger equation. Phys. Rev. E 2002, 66, 056108. [CrossRef] [PubMed]

13. Baleanu, D.; Golmankhaneh, A.K.; Golmankhaneh, A.K. The dual action of fractional multi time Hamilton equations. Int. J. Theor. Phys. 2009, 48, 2558-2569. [CrossRef]

14. Khalil, R.; Al Horani, M.; Yousef, A.; Sababheh, M. A new definition of fractional derivative. J. Comput. Appl. Math. 2014, 264, 65-70. [CrossRef]

15. Abdeljawad, T. On conformable fractional calculus. J. Comput. Appl. Math. 2015, 279, 57-66. [CrossRef]

16. Qian, D.; Li, C.; Agarwal, R.P.; Wong, P.J. Stability analysis of fractional differential system with Riemann-Liouville derivative. Math. Comput. Model. 2010, 52, 862-874. [CrossRef]

17. Almeida, R.; Guzowska, M.; Odzijewicz, T. A remark on local fractional calculus and ordinary derivatives. Open Math. 2016, 14, 1122-1124. [CrossRef]

18. Katugampola, U.N. New approach to a generalized fractional integral. Appl. Math. Comput. 2011, 218, 860-865. [CrossRef]

19. Sene, N. On stability analysis of the fractional nonlinear systems with Hurwitz state matrix. J. Fract. Calc. Appl. 2019, 10, 1-9.

20. Liu, S.; Jiang, W.; Li, X.; Zhou, X.F. Lyapunov stability analysis of fractional nonlinear systems. Appl. Math. Lett. 2016, 51, 13-19. [CrossRef]

21. Souahi, A.; Makhlouf, A.B.; Hammami, M.A. Stability analysis of conformable fractional-order nonlinear systems. Indag. Math. 2017, 28, 1265-1274. [CrossRef]

22. Delavari, H.; Baleanu, D.; Sadati, J. Stability analysis of Caputo fractional-order nonlinear systems revisited. Nonlinear Dyn. 2012, 67, 2433-2439. [CrossRef]

23. Wei, Y.; Chen, Y.; Cheng, S.; Wang, Y. Completeness on the stability criterion of fractional order LTI systems. Fract. Calc. Appl. Anal. 2017, 20, 159-172. [CrossRef]

24. Huang, S.; Wang, B. Stability and stabilization of a class of fractional-order nonlinear systems for $0<\alpha<2$. Nonlinear Dyn. 2016, 2, 973-984.

25. Huang, S.; Wang, B. Asymptotical stability of Riemann-Liouville fractional nonlinear systems. Nonlinear Dyn. 2016, 86, 65-71.

26. Li, M.; Lim, S.C.; Chen, S. Exact Solution of Impulse Response to a Class of Fractional Oscillators and Its Stability. Math. Probl. Eng. 2011, 2011, 657839. [CrossRef]

27. Li, Y.; Chen, Y.; Podlubny, I. Mittag-Leffler stability of fractional order nonlinear dynamic systems. Automatica 2009, 45, 1965-1969. [CrossRef]

28. El-Sayed, A.M.; Arafa, A.A.; Khalil, M.; Hassan, A. A Mathematical Model with Memory for Propagation of Computer Virus Under Human Intervention. Progr. Fract. Differ. Appl. 2016, 2, 105-113. [CrossRef]

29. Priyadharsini, S. Stability Of Fractional Neutral and Integrodifferential Systems. J. Fract. Calc. Appl. 2016, 7, 87-102.

30. Elettreby, M.F.; Tamer Nabil, A.A.A.-R. Dynamical analysis of prey-predator fractional order model. J. Fract. Calc. Appl. 2017, 8, 237-245.

31. Choi, S.K.; Kang, B.; Koo, N. Stability for Caputo fractional differential systems. Abstr. Appl. Anal. 2014, 2014, 631419. [CrossRef]

32. Kilbas, A.; Srivastava, H.; Trujillo, J. Theory and Applications Offractional Differential Equations; Elsevier: New York, NY, USA, 2006.

33. Podlubny, I. Fractional Differential Equations: An Introduction to Fractional Derivatives, Fractional Differential Equations, to Methods of Their Solution and Some of Their Applications; Academic Press: Cambridge, MA, USA, 1998; Volume 198.

34. Miller, K.S.; Samko, S.G. A note on the complete monotonicity of the generalized Mittag-Leffler function. Real Anal. Exch. 1997, 23, 753-755.

35. Li, M. Three classes of fractional oscillators. Symmetry 2018, 10, 40. [CrossRef]

(c) 2018 by the author. Licensee MDPI, Basel, Switzerland. This article is an open access article distributed under the terms and conditions of the Creative Commons Attribution (CC BY) license (http:/ / creativecommons.org/licenses/by/4.0/). 